\title{
Le testament de Jasmin Ledoux Mona Mikaël
}

Maître Renaud toussa, ajusta ses lunettes, balaya du regard l'assemblée attentive et, ouvrant le dossier déposé devant lui, commença d'une voix sourde qu'on ne lui connaissait pas : «Mesdames et messieurs, avant de vous donner lecture du testament de M. Ledoux, je tiens à vous dire que c'est la première fois dans ma très longue carrière que j'ai à m'occuper d'un document pareil. Vous choisirez vous-mêmes l'adjectif dont vous voudrez le qualifier. »

$* * *$

Pourquoi un homme sans biens ferait-il un testament? Que laisse-t-il derrière lui qui profite aux vivants ? Vous allez le savoir, car ce testament-là n'est pas celui de l'homme pauvre que vous avez connu, mais celui d'un riche, très riche héritier dont l'histoire malheureuse en a fait le pauvre homme apparu un matin dans votre petite ville un bâton à la main, une écritoire sous le bras. Cette histoire, je vous la livre afin que chacun puisse s'y voir avec mes yeux et comprendre quel rôle il a pu y jouer.

Je suis le fils aîné d'un grand industriel, Armand Langevin, qui avait fait fortune dans l'acier pendant la Deuxième Guerre. À ma naissance, il m'appela Hector alors que ma mère aurait voulu Jasmin. Il y avait entre ces deux noms, l'un puissant et l'autre parfumé, toute la distance qui séparait l'ambition paternelle de la suave douceur de ma mère. J'ai porté le premier jusqu'à la mort de mon père et l'autre par la suite, réalisant ainsi entre mes parents comme un accord posthume.

L'auteur de mes jours, me tenant à bout de bras, avait dit d'une voix forte : "Hector Langevin, nous allons faire ensemble la grande conquête du monde ! » Mais un ange fatigué avait mis sur mon front l'Étoile du Rêveur au lieu de celle, avide et commerçante, qui eut peut-être fait mon bonheur. Je devrais dire plutôt le bonheur de mon père, car c'est à lui d'abord que le temps réservait - comme une punition seulement retardée - la déception de l'un de ses plus beaux espoirs.

On m’appelait « le doux », mais pour lui, j'étais « le mou ».

La naissance de mon frère réalisa son rêve d'un successeur plus digne. J'étais aussi timide et effacé que Richard était fort et ambitieux, et quoique j'eusse sur lui l'avantage de la taille, il s'imposait partout comme le chef et l'aîné. Le favori aussi. Mais j'avais pour mon frère un 
attachement si fort, que ni les préférences dont il était l'objet, ni les mille vexations dont m'abreuvait mon père ne pouvaient l'affaiblir.

Richard était le seul qui pouvait tenir tête à celui que le monde surnommait l'Homme d'Acier, et lui reprocher en face ses injustes brimades. Notre chère mère, à qui je ressemblais, avait toujours paru quelque peu étonnée d'avoir donné la vie à un être aussi différent d'elle. Mais qu'importe ? Armand avait son dauphin et cela seul comptait. De plus, les garçons s'entendaient, et cela aussi comptait.

Mais, hélas ! rien ne dure ici-bas.

Pour tout le sang versé par les armes sorties de ses usines d'acier, mon père allait sentir le boulet du Destin s'abattre sur son sang, et donc sur sa famille. Ma mère fut la première à partir, terrassée par une fièvre qui, d'un large coup de faux, décima notre ville. Cette fièvre meurtrière fut le seul point commun, également posthume, qu'elle eût jamais avec son second fils, car celleci emporta aussi notre précieux Richard. Il avait dix-neuf ans.

Mon père et moi, nous restâmes seuls ; je ne dis pas «ensemble », car nous l'étions si peu... Plongés dans la même douleur mais séparés par elle, nous étions deux îlots de tristesse dans l'océan du monde. Nous nous parlions rarement et comme à contrecœur jusqu'au jour où mon père, qui baissait à vue d'œil, m'appela dans sa chambre. Je fus si effrayé par son aspect hagard, que mon premier mouvement en fut un de recul ; mais il me commanda de l'écouter sans bruit.

À mon immense surprise, il me saisit la main et me demanda pardon.

«Je suis un homme cassé, Hector, dit-il ensuite d'une voix éteinte. La grande conquête du monde, tu la feras sans moi et pas par mon acier, mais par ta belle douceur que j'ai trop longtemps prise pour de la mollesse. Heureux les doux, car ils possèderont la terre ; je l'ai compris trop tard. Tout ce que je peux t'offrir en réparation, c'est un plan pour que ton argent ne fasse pas ton malheur. » Dès qu'il m'eut dit son plan, l'Homme d'Acier expira.

Maintenant que je m'apprête à le rejoindre bientôt, je l'absous entièrement de ses duretés passées, car c'est grâce à ce plan si simple et ingénieux que j'ai pu vivre en paix sans le venin du doute, cet insidieux poison qui fait que tant de riches se demanderont toujours si ceux qui s'approchent d'eux recherchent leur amitié ou plutôt leur argent.

J'étais désormais seul au milieu de la terre. 
Bien que mes chers défunts y fussent tous enterrés, je quittai le pays, chassé par le chagrin et par les convoitises que lisait dans les yeux des vautours alléchés le juteux milliardaire que j'étais devenu... L'idée d'être adulé à cause de mon argent me répugnait tellement, que j' aurais volontiers déposé cette fortune aux pieds d'un vieux clochard affamé et crasseux, certain que son sourire serait vraiment sincère.

J'avais alors vingt-deux ans, mais vieilli par la peine, j'en paraissais quarante.

En prenant le nom de Jasmin qu'avait choisi ma mère à ma naissance, j'entrai pour ainsi dire dans son orbite aimante, ayant comme déposé le passé douloureux d'Hector Langevin. Muni de mon bâton et d'un léger bagage, j'étais bien résolu à vivre modestement afin de pouvoir jouir de cette belle liberté des gens privilégiés qui n'ont et ne sont rien. Aucune adaptation ne me fut nécessaire pour passer de l'enfant riche à l'homme des grands chemins.

Le métier d'écrivain ambulant me permit de circuler sans avoir à livrer aucun de mes secrets.

Je vécus en bohème pendant toute une année, allant de ville en ville dans ma peau de quidam, dormant sous les étoiles et savourant pleinement dans chacune de mes fibres cette longue bouffée d'air frais... Mais l'oiseau de passage, fatigué de n'être plus personne pour personne, se posa un matin sur un rocher rugueux surplombant l'océan, aux abords de la ville de M... La poésie du lieu et sa beauté sauvage m'incitèrent à rester un temps dans le pays.

Ce large rocher plat s'avançant en plongeoir juste au-dessus des vagues et donnant l'impression qu'on était en pleine mer sur une barque immobile allait vite devenir mon endroit préféré. Je m'y rendais chaque jour, bientôt rejoint par deux ou trois casquettes et quelques châles flottants que mon personnage intriguait. Il en vint d'autres encore. Il en vint tout une foule. En me voyant ainsi entouré, je plantai dans ce sol mon lourd bâton d'errant pour laisser s'amasser sur ma pierre vagabonde quelques bonnes couches de mousse.

Ma journée terminée - même un scribe ambulant a ses heures de travail ! - , je courais au Rocher, certain d'y être seul pendant une heure au moins avant ce que j'appelais en souriant « l'invasion ». J'y méditais, tranquille, ma moisson quotidienne de rencontres, d'impressions, nourri de mille joies simples, de sourires, de regards, de visages expressifs qui me parlaient au cœur. Celui-ci me rappelait ma mère, cet autre mon frère Richard, mais pas une seule figure qui évoquât mon père... 
Un matin, cependant, une exquise petite femme ridée comme une vieille pomme et qui m'avait pris en amitié vint me dicter une lettre. Elle remercia, paya et dit en me prenant le visage dans ses mains : « Tu portes bien ton nom, mon aimable Jasmin. Heureux les doux, car ils possèderont la terre ». L'écho de ces paroles prononcées par mon père juste avant de mourir creva l'outre des larmes si longtemps retenues... Qu'il était bon de pleurer dans les bras de cette femme !

Certes, j'aurais pu le faire dans des bras bien plus jeunes qui se seraient tendus d'euxmêmes vers moi ; mais ceux dont je rêvais ne me recherchaient pas. Combien de robes flottant au vent frais de la mer, de chevelures dansantes, de formes ondoyantes m'auraient vite entraîné dans un soyeux vertige si mon cœur n'avait pas déjà choisi sa reine... Mais la peur du rejet paralysait ma langue et je n'osais me déclarer, retardant constamment le moment de l'aveu.

Si grande était mon indécision, que je n'en dormais plus. Mais voilà que le destin, dressant une fois de plus son spectre sur ma route, trancha net la question d'un terrible coup de glaive ! Bien que je ne ressentisse qu'une très grande fatigue, le médecin m'informa qu'il me restait seulement quelques semaines à vivre... Comme mon père, je m'en allais d'une affreuse leucémie. J'avais juste trente ans.

Pourquoi ce coup de poignard dans mon cœur encore jeune?

Quel pouvoir dictateur exigeait que je fasse le deuil de ce qu'un homme possède de plus précieux, l'amour et la vie ? Ma détresse, explosant en révolte, trouva dans la fureur des éléments une sorte d'exutoire. La tempête et mon âme rugissaient en même temps. Puis vint l'acceptation. Après avoir maudit le destin qui m'emportait si vite, j'en vins à le bénir d'avoir frappé son coup avant que j'aie parlé à la femme que j'aimais.

Il ne fallait pas qu'elle sache pour ne pas la troubler, car ce sont là des choses qu'un souffle peut froisser.

Cependant, je pensais très souvent à mon père à cause du fameux plan qu'il avait établi pour que je puisse avoir accès à son argent tout en menant la vie simple que je voulais. Le bâton de voyage qui ne me quittait pas était un tube d'acier confectionné par lui, recouvert de faux bois, avec à l'intérieur trois longs compartiments superposés. Dans l'un d'eux, je pouvais glisser plusieurs rouleaux de billets que j'allais retirer à une certaine banque.

Une fois tous les trois mois, je m'habillais « en riche » pour faire cette transaction dans une ville éloignée et, au retour comme à l'aller, je changeais de vêtements à mi-chemin dans les bois. 
Grâce aux rencontres sur le Rocher, j'étais très au courant des besoins de chacun et pus ainsi, pendant sept ans, éprouver la joie pure d'offrir une aide discrète. Aux uns, je disais que c'était un don de leur saint préféré ; aux autres, qu'un oiseau avait « déposé ça » en passant sur leur toit; à la plupart, rien.

Mais c'étaient de petites sommes proportionnées à mon revenu de scribe, pour ne pas éveiller les soupçons.

Aux autres compartiments de mon bâton d'acier, je ne devais toucher qu'en cas de catastrophe. Ils contenaient, l'un des pièces d'or pur empilées dans le tube, et l'autre des diamants incrustés dans de la glaise. Je pouvais ainsi garder une partie de ma fortune sous la main en tout temps, personne ne se doutant qu'un bâton de nomade eût pu servir d'écrin à tant de choses précieuses...

Saurez-vous, gens de M..., ce que votre amitié, cette douce manne du cœur, a donné au pèlerin qui déposa chez vous son bâton, et combien ce bâton vous appartient de droit ? S'il est vrai que l'amitié ne peut se monnayer, je n'ai que de l'argent pour la récompenser et le trésor que je laisse n'est en rien comparable à celui que j' emporte. Qu'aucun de vous n'aille croire qu'il me doit quelque chose, car ma dette envers vous est incommensurable.

\section{$* * *$}

Maître Renaud se tut, n’osant lever la tête. Il lui restait à lire le corps du testament, avec la répartition des biens si avidement attendue d'ordinaire. Mais les gorges serrées et les yeux ruisselants imploraient en silence un temps d'arrêt, une pause. Il n'était pas sûr lui-même de pouvoir continuer. Alors, il se leva, éteignit le micro et regarda lentement la petite ville désolée massée en foules compactes sur la place, dans les rues et jusque sur le Rocher. 